\title{
A Neural Based Experimental Fire-Outbreak Detection System for Urban Centres
}

\author{
Agaji Iorshase1, Shangbum F. Caleb ${ }^{2}$ \\ ${ }^{1}$ Department of Mathematics/Statistics/Computer Science, University of Agriculture, Makurdi, Nigeria \\ ${ }^{2}$ Department of Computer Science, Nigerian Army Institute of Technology \& Environmental Studies, Makurdi, \\ Nigeria \\ Email: sasemiks@gmail.com, ior.agaji@uam.edu.ng
}

Received 6 January 2016; accepted 20 March 2016; published 23 March 2016

Copyright $(\subset 2016$ by authors and Scientific Research Publishing Inc.

This work is licensed under the Creative Commons Attribution International License (CC BY).

http://creativecommons.org/licenses/by/4.0/

c) (i) Open Access

\begin{abstract}
Incessant fire-outbreak in urban settlements has remained intractable especially in developing countries like Nigeria. This is often characterized by grave socio-economic aftermath effects. Urban fire outbreak in Nigerian cities has been on increase in recent times. The major problem faced by fire fighters in Nigerian urban centres is that there are no mechanisms to detect fire outbreaks early enough to save lives and properties. They often rely on calls made by neighbours or occupants when an outbreak occurs and this accounts for the delay in fighting fire outbreaks. This work uses Artificial Neural Networks (ANN) with backpropagation method to detect the occurrence of urban fires. The method uses smoke density, room temperature and cooking gas concentration as inputs. The work was implemented using Java programming language and results showed that it detected the occurrence of urban fires with reasonable accuracy. The work is recommended for use to minimize the effect of urban fire outbreak.
\end{abstract}

\section{Keywords}

Fire-Outbreak Detection, Neural Network, Urban Fires, Backpropagation, Sigmoid Transfer Function, Fire Alert, Temperature, Smoke Density, Cooking Gas Concentration, Weights

\section{Introduction}

Inferno catastrophes have remained a problem of contemporary urban society. Scores of lives and billions of naira worth of goods and property have been lost to urban fires. Raze of residential buildings, office complexes and market structures by fire have become a common calamity in urban centers and suburbs in Nigeria. The degree of the impact of fire-outbreak on the urban centres is enormous and most times difficult to quantify. The 
smoke, heat as well as the bad odour from such infernos normally constitutes health hazards. According to [1] the emission of carbon and other gases into the atmosphere results in global warming that inevitably causes ecological upheavals. Urban fire outbreak has not only led to loss of lives, but has left many homeless and with many degrees of disability. Survivors of fire disasters are widely found to be helpless amidst loss of livelihood [2]. Fire aftereffects have spontaneously wrecked havoc spanning from temporal frustration, to temporal or permanent reduction of health and wealth-related quality of life, to post traumatic stress disorder, feeling jittery and jerky, avoiding reminders of the fire nightmares [3]. The major task of this work is the development of urban fire prediction system based on ANN with the aim of curbing the damages and calamities caused by the occurrence of urban fires.

\section{Related Literature}

Urban buildings are inevitably vulnerable to fire and the timely detection of fires in urban buildings is a common global challenge in safeguarding metropolitan habitats, fortifying lives and property and averting consequent economic damages. Correct and reliable domestic, office and market fires detection results are essential for decision support in planning of fire fighting. Many methodologies have been developed in the past for detecting and predicting forest wild fires using ANN. This is affirmed by [4] that most existing work in fire occurrence prediction focuses on prediction of wildfires in forests and those caused by volcanic eruptions. In urban centers alarms are mostly used for the detection of fire outbreak.

A fire risk ignition scheme that can be an integral component of a quantitative fire danger rating system was presented by [5] for Lesvos Island Greece. The scheme used meteorological data to estimate the geo-spatial fire risk regardless of fire causes or expected burned area. The output of the scheme is Fire Ignition Index which is based on Fire Weather Index, Fire Hazard Index and Fire Risk Index.

A framework for the development of forest fire prediction in Lebanon using decision tree algorithm and neural networks was presented by [6]. Their prediction was anchored on four meteorological attributes namely; temperature, relative humidity, wind speed and daily precipitation. In their framework, they adopted neural networks with different algorithms and comparatively showed that networks with only two inputs (temperature and relative temperature) produced better results with less MSE than one with the 4-inputs.

A neural-networks-based approach to the problem of predicting forest fires using multilayer perceptron whose number and size of hidden layers can be heuristically determined for each application using its available data was put forward by [7]. This approach utilizes forest fire data from Portuguese Montesinho Natural Park and used backpropagation algorithm to train the neural network.

A multi-agent Decision Support System for Forest Fire was presented by [8]. Their system called Forest Fire Decision Support System (FOFDESS) did the fire danger rating by predicting the forest fire. It was also used in the approximation of fire spread speed and quick detection of a started fire. They used some data fusion algorithms such as Artificial Neural Network (ANN), Naive Bayes Classifier (NBC), Fuzzy Switching (FS) and image processing for predicting the forest fire.

A probit model was used by [9] to assess the chance of fires in the English Peak District at different times of the year, days of the week under various weather conditions. They observed that the likelihood of fire increases with maximum temperature. Their model back-predicts fires during a hot dry summer.

A model for estimating the outbreak of fires using auto-regressive process to obtain the predicted number of fires during a day was presented by [10]. They used Garcia in Spain as a testing area. Their model has no limitation on the burnt area and as such incorporated parameters that may not be needed in the prediction of urban fires.

An overview on how forest fire detection, emission estimation and fire risk prediction in China was carried out using satellite imagery, climate data and various simulation models over the past three years was presented by [11] They reported the use of data acquired by means of numerous satellites like ENVISAT, MODIS. To improve fire risk modeling they reported that real-time meteorological data such as humidity, wind speed and direction were used as input for the prediction of forest fire occurrence and its behavior.

An assessment of three systems used for the detection of wide fires in Australia was carried out by [12]. The systems were EYEfi, Fire Watch and Forest Watch. The system was all based on image analysis from sensors mounted on fixed towers. While the two of the systems provided supervised automatic detection, EYEfi, one of the systems did manual target and reported some fires. The major problem with these systems was that the cameras used were both slower and less reliable than trained human observer. 
The studies that probed the background in Geostationary Operational Environmental Satellite (GOES) 15 data was carried out by [13]. Also probed was the sensitivity of a fire detection satellite in geosynchronous orbit. They suggested a number of algorithms that can reduce false alarms. They also proposed the framework for a geosynchronous satellite that was able to detect heat from early and small fires and yielded minute-scale detection times. The major problem with their method is the lack of automatic detection of fires. For some fires a delay of up to a minute could cause a major catastrophe.

Fire detection algorithm in video sequences on wireless sensor network was proposed by [14]. Their algorithm processed visual information acquired through static camera. Their algorithm was incorporated in CCTV surveillance system. Their algorithm detected foreground of video sequences, color detection in RBG space and was able to eliminate all objects that do not fulfill color requirements without fire-like objects.

The system of monitoring fire based on information fusion technology using Dempster-Shafer evidence theory was built by [15]. Their system avoided the failure of monitoring data effectively and dealt with the conflicting evidence from the multi-sensor and greatly improved the reliability fire warning.

Most of the literature reviewed dwell on the prediction of wild or forest fires with little or no attention to the detection of urban fires. Detection methods based on cameras and videos are slower than human observers in detecting fire outbreaks.

\section{Methodology}

The method used in detecting fire-outbreak in this work is ANN and Makurdi, a very fast growing town in North Central Nigeria, is used as a testing area. The area is selected because of the increased frequency of fire outbreak in the town with the attendance loss of lives and property in recent times. The current method of fire outbreak detection in urban buildings in Makurdi using alarms has high rate of false alerts and also alerts only the occupants of the building but cannot alert fire fighters who may be found outside the building. The false alerts are due to lack self learning and intelligence on the part of the alarms. The alarms are also only effective if the occupants are around. This requirement that the occupants be present may only hold if one is currently in his/her house. For offices and markets where there are clearly defined closing and working hours if a fire occurs during closing hours remedy may be delayed.

The proposed system, after training, detects fire in urban buildings based on real time data. The detection method of the system is simplified using the following steps.

(i) Create a Three-layer Perceptron Neural Network (TLPNN).

(ii) Acquire Fire-outbreak data based on smoke density, temperature and cooking gas concentration of burned urban buildings before being engulf by fire are acquired;

(iii) Normalize the fire data into a homogeneous data interval $[0,1]$.

(iv) Configure the Three-layer Perceptron Network for fire detection by training and validating the network with training and testing data respectively.

(v) Set in real data for the actual detection in which fire is detected if the output is 1 (one) and no fire is presented if output is 0 (zero).

(vi) Alert firemen and occupants of the building if fire is detected.

This architecture of the system is shown in Figure 1.

The architecture is made up of two components. The first component is the training environment. Randomly generated data that bears semblance to actual data is acquired through random number generator. The data is used normalized and fed into the NN training section. After training the resultant NN is tested and validated. The validated NN is the second component and is used for the detection of fires in urban centers. This architecture is characterized by a network of simple processing units called neurons connected by links as shown in Figure 2. The network consists of three layers: the input layer, the hidden layer and the output layer. The input layer has three neurons which are fed up with three observations $s_{1}, s_{2}, \ldots, s_{n-1}, s_{n}, t_{1}, t_{2}, \ldots, t_{n-1}, t_{n}$ and $c_{1}$, $c_{2}, \ldots, c_{n-1}, c_{n}$ representing the smoke density, room temperature and cooking gas concentration variables respectively. The hidden layer is made up of six units while the output layer is made up of one unit. The hidden layer outputs are then linked as input to the output layer using sigmoid function to produce the output, $\mathrm{y}$.

\section{Design of the System}

The design of the system commences with the specification of the activities carried out by the system. The 


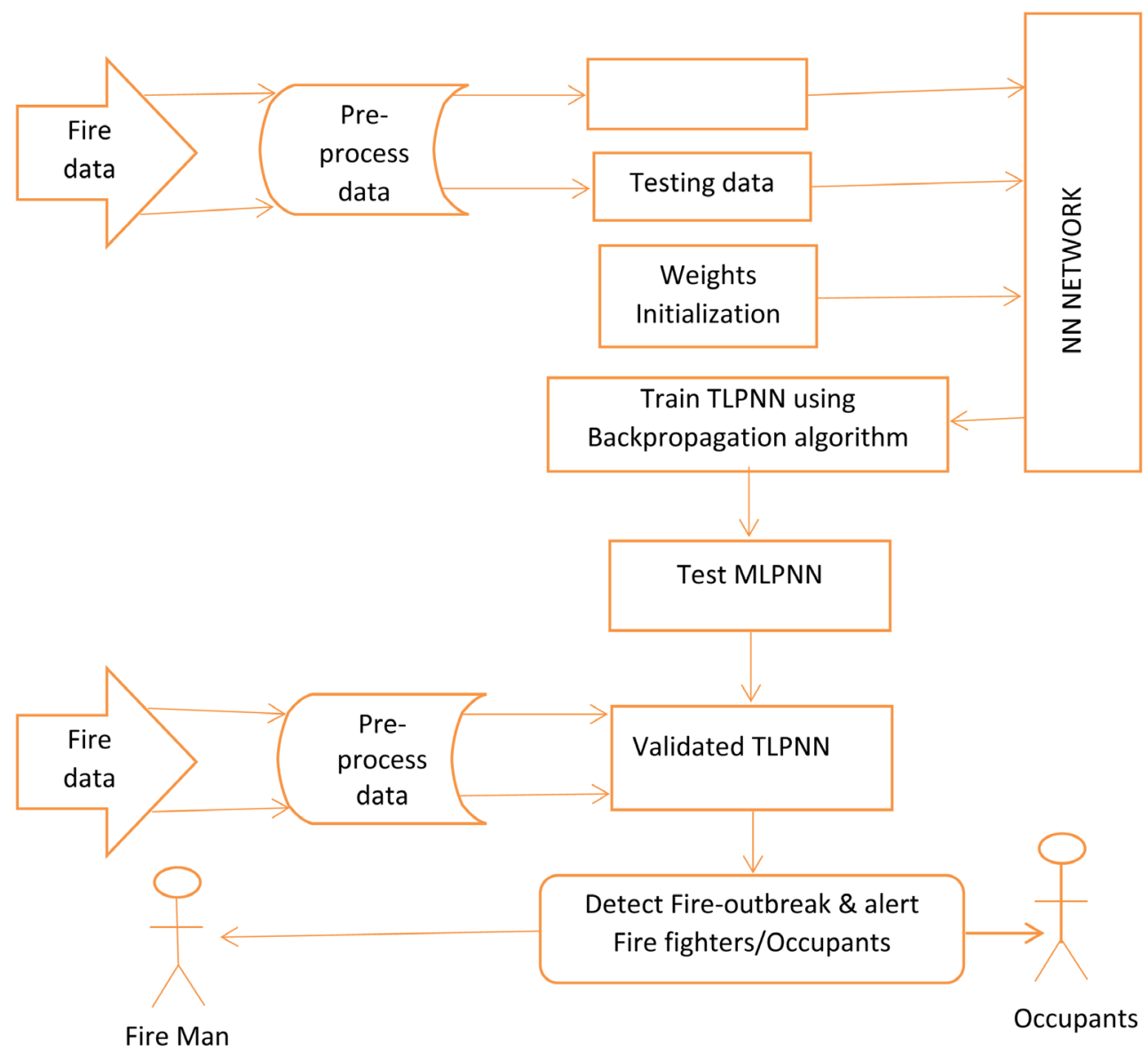

Figure 1. Architectural model for the proposed system.

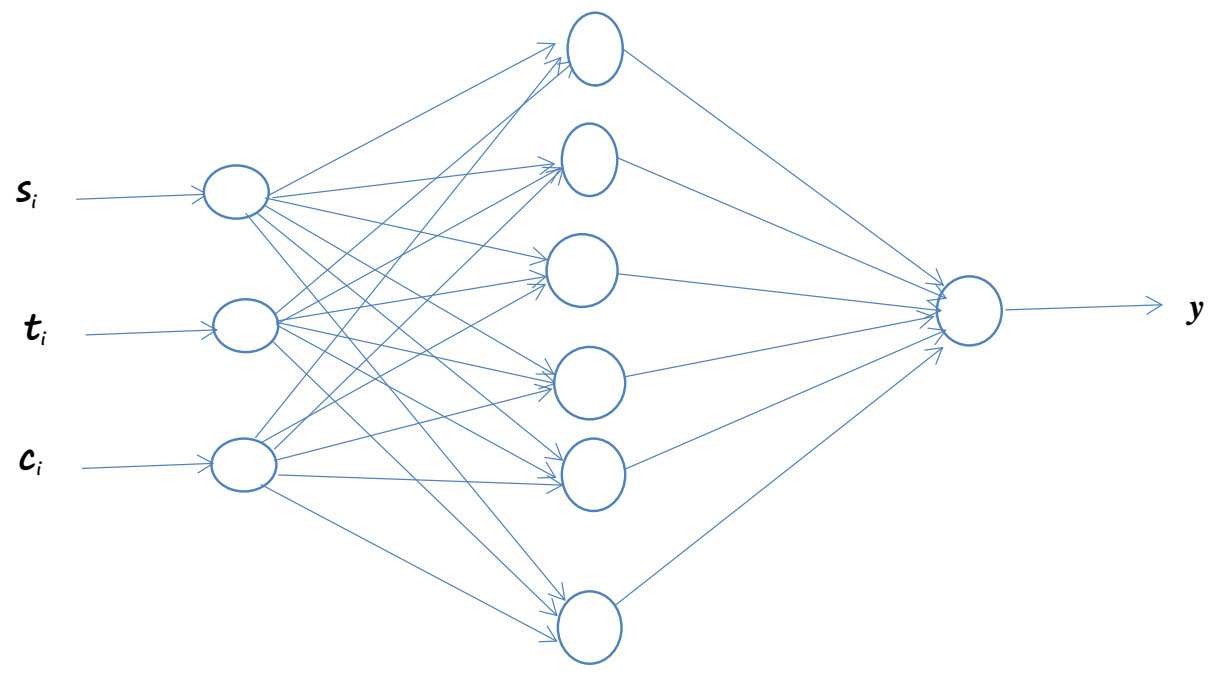

Figure 2. A network of Neurons.

activities are shown in Figure 3. Three inputs units and one output unit are used in this study because only three inputs are required into the system and a single detection output. The output represents the two states of detection namely: Fire-outbreak $(y=1)$ and No Fire-outbreak $(y=0)$. The value of the network output, $y$ is determined as expressed in Equation (1.1). 


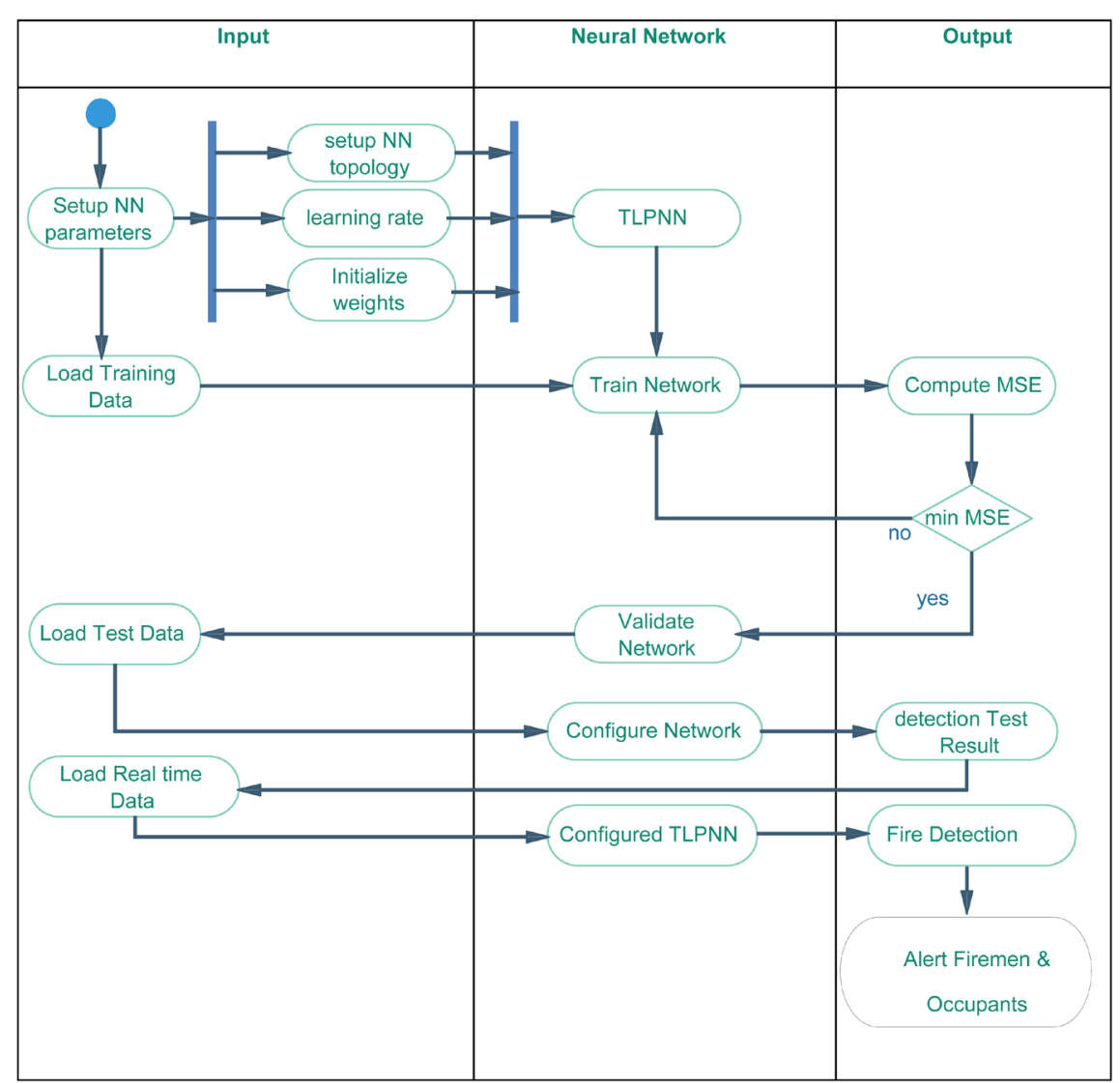

Figure 3. Activity diagram for NN fire-outbreak detection system.

$$
y=\left\{\begin{array}{l}
1, \text { if } 0.5 \leq f(\varphi) \leq 1 \\
0 \text { otherwise }
\end{array}\right.
$$

where $f(\varphi)$ is the sigmoid function and $\varphi$ represents the weighted sum of inputs

One hidden layer is used because of its ability to effectively approximate nonlinear and continuous functions. It also prevents the adverse effect of large number of hidden layers on training times gradient based learning. The choice of the number of hidden neurons is very crucial to the effectiveness of a neural network. A single hidden layer also enables the network growing strategy.

Fire is detected to occur if the final network output is 1 (one) and an alert is sent to fire fighters and occupants. Figure 3 shows the workflow of activities for the proposed system. In the figure, a three-layered neural network is established after receiving the setup parameters and the network training is performed after loading the training data. If the mean square error is sufficiently low, the system is validated and ready to accept real data. The configured or validated network can now be used for the detection of urban fires. An alert is sent to both firemen and the owner of the building if the network output is one (1). The sequence diagram for the system is shown in Figure 4.

Figure 4 shows the interaction between the system processes and its environment. The environment is made up of the administrator and users. The administrator sets up the NN system, carries out training and validation of the system before deploying it for users. The users who are the owners of buildings and the fire men receives alerts from the system through the process called the TPLNN output.

Figure 5 shows the flowchart of the proposed system. The flowchart has two components separated by the first decision box. The first component is in charge of training and the second component is in charge of detecting the occurrence of urban fires. In the first component the system is initialized by creating a neural network, initializing weights and accepting normalized inputs for forward propagation. The error is continuously back 


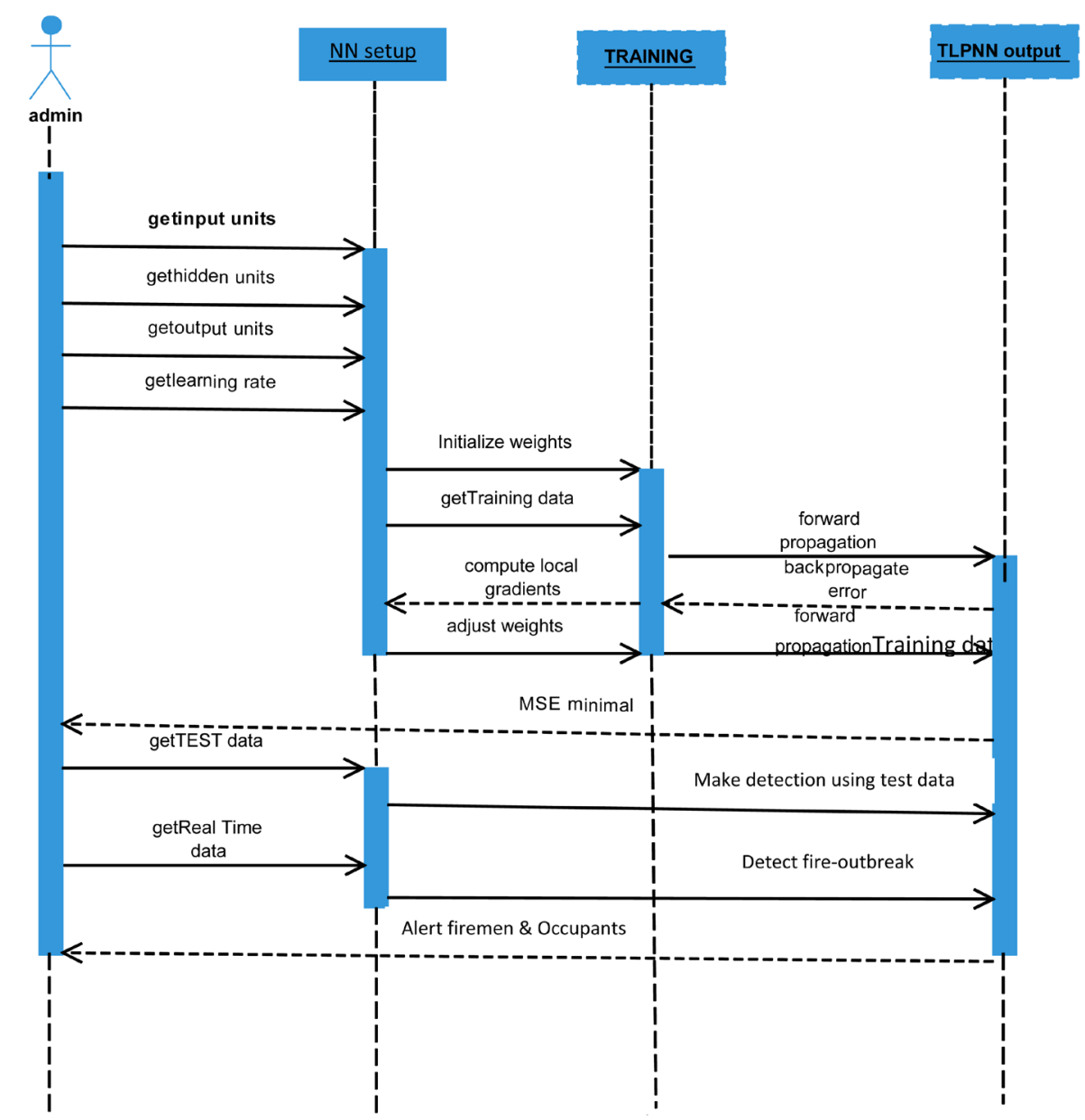

Figure 4. A sequence diagram for the ANN fire detection system.

propagated if it is not optimal. This component is validated by testing the network with the test data. In the second component real time data is inputted into the network for actual fire detection. If the system is already trained the real time data is directly passed to the system.

\section{Implementation, Results and Discussion}

The observational data of urban building fire outbreaks were generated based on smoke densities, room temperature and gas concentration attributes. The data of size 50 was randomly generated using Java random number generators. The data was normalized to have semblance to actual fire data and used in the training the system using the backpropagation training algorithm. Java programming language was used for the simulation. The 3-6-1 topology was adopted after a series of experiments with other topologies. This topology gave the most suitable results. This topology has three input neurons represented by the three input facilities, six units in the hidden layer and one output layer with one unit. The various experiments carried out as well their results are presented as follows.

\section{Experiments}

Five (5) experiments were carried out. Five different sets of randomly generated fire data with three numerical values corresponding to temperature, smoke density and cooking gas concentration were used. The input values as well as the corresponding outputs are shown in Table 1.

The first column in the table contains the number of datasets. Five different datasets were used. Each dataset contains three normalized values representing temperature (temp), smoke density (smokeden) and cooking gas 


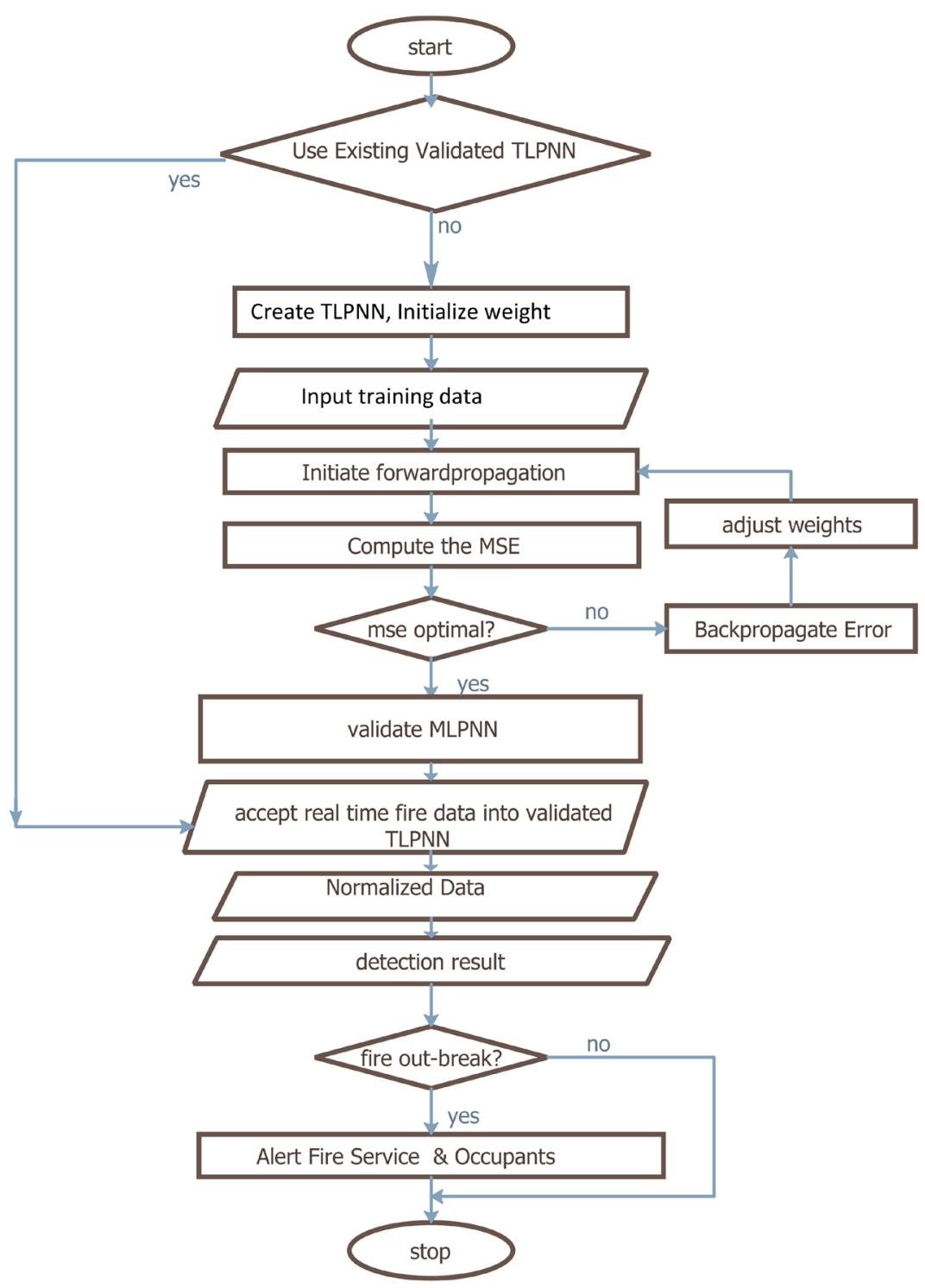

Figure 5. System flowchart.

Table 1. Input values and their corresponding results.

\begin{tabular}{ccccc}
\hline Dataset & Temp & Smokeden & gascon & Result \\
\hline 1 & 0.81255 & 0.89899 & 0.633333 & 0.988018 \\
2 & 0.1125 & 0.030303 & 0.188889 & 0.038202 \\
3 & 0.475 & 0.367347 & 0.633333 & 0.590639348 \\
4 & 0.375 & 0.479592 & 0.644444 & 0.626675804 \\
5 & 0.025 & 0.020408 & 0.1 & 0.000224639 \\
\hline
\end{tabular}

concentration (gascon). The values were normalized to lie between zero and one. The normalization was done as shown in Equation (4.1).

$$
a=\frac{(x-\operatorname{Min}(x))}{\operatorname{Max}(x)-\operatorname{Min}(x)}
$$


In Equation (4.1), a represents the normalized value, $x$ represents an input value to be normalized, min(x) represents the minimum input allowed for the system and max (x) represents the maximum input allowed for the system. For example, a temperature of $75^{\circ} \mathrm{C}$ was normalized to be 0.6875 using the minimum temperature of $20^{\circ} \mathrm{C}$ and maximum temperature of $100^{\circ} \mathrm{C}$. The minimum temperature was chosen to reflect the actual temperature of Makurdi whose temperature has never fallen below this value. Column four represents the results of the system. For fire to occur, the output must be greater or equal to 0.5 . Values below 0.5 indicate no fire. In the table the use of dataset 1 , dataset 3 and dataset 4 indicated fire since each of the output was greater or equal to 0.5 , while the use of dataset 2 and dataset 5 indicated no fire since each of the output was less than 0.5 .

\section{Conclusion and Recommendation}

The present work proposed a neural based system for detection of urban fire. The work used three input values made up of temperature, smoke density and cooking gas concentration. The system indicated the occurrence of fire if the output was close to one. The work was implemented using Java programming language and results showed that if detected the occurrence of urban fires with reasonable accuracy. The system is recommended for use in Nigeria cities because it avoids false fire alarms.

\section{References}

[1] Stojanova, D., Andrej, K., Peter, O., Bernard, Z. and Saso, D. (2012) Estimating the Risk of Fire Outbreaks in the Natural Environment. Data Mining and Knowledge Discovery, 24, 411-442.

http://www-ai.ijs.si/SasoDzeroski/pdfs/2012/2012-StojanovaEtAl-DAMI_2.pdf

http://dx.doi.org/10.1007/s10618-011-0213-2

[2] Machilis, G.E., Kaplan, A.B., Tuler, S.P., Bagby, K.A. and McKendry, J.E. (2002) Burning Questions: A Social Science Research Plan for Federal Wildland Fire Management. University of Idaho, Moscow, Idaho. https://books.google.com.ng/books?id=obIrAQAAMAAJ\&printsec=frontcover\#v=onepage\&q\&f=false

[3] Nyamadzawo, G., Gwenzi, W., Kanda, A., Kundhlande, A. and Masona, C. (2013) Understanding the Causes, SocioEconomic and Environmental Impacts, and Management of Veld Fires in Tropical Zimbabwe. Fire Science Reviews. Springer Berlin Heidelberg. http://download.springer.com/static/pdf/11/art\%253A10.1186\%252F2193-0414-2-2.pdf http://dx.doi.org/10.1186/2193-0414-2-2

[4] Divya, T.L. and Vijayalakshmi, M.N. (2013) Development of Frame Work for Prediction of Forest Fire and Fire Spread Direction Using Image Mining. International Journal of Advanced Research in Computer and Communication Engineering, 2, 4878-4882. http://www.ijarcce.com/upload/2013/december/IJARCCE9B_a_divya_Development.pdf

[5] Vasilakos, C., Kalabokidis, K., Hatzopoulos, J., Kallos, G. and Matsinos, Y. (2007) Integrating New Methods and Tools in Fire Danger Rating. International Journal of Wildland Fire, 16, 306-316 http://forecast.uoa.gr/Publications/72_Vasilakos_Int_J_Wildf.pdf http://dx.doi.org/10.1071/WF05091

[6] Karouni, A., Daya, B. and Chauvet, P. (2014) Applying Decision Tree Algorithm and Neural Networks to Predict Forest Fires in Lebanon. Journal of Theoretical and Applied Information Technology, 63, 282-291. http://www.jatit.org/volumes/Vol63No2/6Vol63No2.pdf

[7] Safi, A. and Bouroumi, A. (2013) Prediction of Forest Fires Using Artificial Neural Networks. Applied Mathematical Sciences, 7, 271-286. http://www.m-hikari.com/ams/ams-2013/ams-5-8-2013/safiAMS5-8-2013.pdf

[8] Çetin, E. and Yusuf, S. (2011) A Data Fusion Framework with Novel Hybrid Algorithm for Multi-Agent Decision Support System for Forest Fire. Expert Systems with Applications, 38, 9225-9236. http://isiarticles.com/bundles/Article/pre/pdf/5704.pdf

[9] Albertson, K., Aylen, J., Cavan, G. and McMorrow (2009) Forecasting the Outbreak of Mooreland Wildfires in the English Peak District. Journal of Environmental Management, 90, 2642-2651. http://www.sciencedirect.com/science/article/pii/S0301479709000498

[10] Diez, E.L.G., Soriano, L.R. and Davila, F.D.P. (1993) An Objective Forecasting model for the Daily Outbreak of Forest Fires Based on Meteorological Consideration. Journal of Applied Meteorology, 33, 519-526. http://journals.ametsoc.org/doi/pdf/10.1175/1520-0450\%281994\%29033\%3C0519\%3AAOFMFT\%3E2.0.CO\%3B2 http://dx.doi.org/10.1175/1520-0450(1994)033<0519:AOFMFT>2.0.CO;2

[11] Zhang, J., Yao, F., Liu, C., Yang, L. and Boken, V.K. (2011) Detection, Emission Estimation and Risk Prediction of Forest Fires in China Using Satellite Sensors and Simulation Models in the Past three Decades-An Overview. International Journal of Environmental Research and Public Health, 8, 3156-3178. www.mdi.com/Jpurnal/Ijerph http://dx.doi.org/10.3390/ijerph8083156 
[12] Mattews, S., Sullivan, A., Gould, J., Hurley, R., Ellis, P. and Larmour, J. (2010) Evaluation of Three Fire Detection Systems. BushFire Cooperative Research Centre.

https://www.ag.gov.au/Publications/Documents/RemoteFireDetectionTrialsEvaluationofThreeFireDetectionSystems/Fi reDetectionPublicReport.pdf

[13] Pennypacker, C.R., Jakubowski, M.K., Kelly, M., Lampton, M., Schmidt, C., Stephens, S. and Tripp, R. (2013) GUEGO-Fire Urgency Estimation in Geosynchronous Orbit-A Proposed Early-Warning Fire Detection System. Remote Sensing, 5, 5173-5192. www.mdi.com/journal/remotesensing http://dx.doi.org/10.3390/rs5105173

[14] Kim, Y., Kim, A. and Jeong, H. (2014) RBG Color Model Based the Fire Detection Algorithm in Video Sequences on Wireless Sensor Network. International Journal of Distributed Sensor Network, 2014, Article ID: 923609. http://dx.doi.org/10.1155/2014/923609

[15] Ding, Q., Peng, Z., Liu, T. and Tong, Q. (2014) Multi-Sensor Building Alarm System with Information Fusion Technology on D-S Evidence Theory. Algorithms, 7, 523-537. www.mdpi.com/journl/algorithms http://dx.doi.org/10.3390/a7040523 